\title{
Isolation of Neuroprotective Anthocyanins from Black Chokeberry (Aronia melanocarpa) against Amyloid- $\beta$-Induced Cognitive Impairment
}

\author{
Haichao Wen ${ }^{1,2}$, Hui Cui ${ }^{1,2}$, Hehe Tian ${ }^{1,2}$, Xiaoxu Zhang ${ }^{2}$, Liyan Ma ${ }^{2,3}$, Charles Ramassamy 4 \\ and Jingming $\mathrm{Li}^{1,2, *}$ \\ 1 Center for Viticulture and Enology, College of Food Science and Nutritional Engineering, \\ China Agricultural University, Beijing 100083, China; wenhc@cau.edu.cn (H.W.); cuihuidaisy@163.com (H.C.); \\ tianhehe@cau.edu.cn (H.T.) \\ 2 College of Food Science and Nutritional Engineering, China Agricultural University, Beijing 100083, China; \\ zxxjoypeace@foxmail.com (X.Z.); lyma1203@cau.edu.cn (L.M.) \\ 3 Supervision, Inspection \& Testing Center for Agricultural Products Quality, Ministry of Agriculture, \\ Beijing 100083, China \\ 4 Institut National de la Recherche Scientifique-Institut Armand Frappier, Laval, QC H7V 1B7, Canada; \\ Ramassamy@iaf.inrs.cn \\ * Correspondence: lijingming@cau.edu.cn; Tel.: +86-010-62737039; Fax: +86-010-62738658
}

\section{check for}

updates

Citation: Wen, H.; Cui, H.; Tian, H.; Zhang, X.; Ma, L.; Ramassamy, C.; Li, J. Isolation of Neuroprotective Anthocyanins from Black Chokeberry (Aronia melanocarpa) against Amyloid- $\beta$-Induced Cognitive Impairment. Foods 2021, 10, 63. https://doi.org/10.3390/ foods10010063

Received: 16 November 2020 Accepted: 24 December 2020 Published: 29 December 2020

Publisher's Note: MDPI stays neutral with regard to jurisdictional clai$\mathrm{ms}$ in published maps and institutional affiliations.

Copyright: $(2020$ by the authors. Licensee MDPI, Basel, Switzerland. This article is an open access article distributed under the terms and conditions of the Creative Commons Attribution (CC BY) license (https:// creativecommons.org/licenses/by/ $4.0 /)$.

\begin{abstract}
Black chokeberry (Aronia melanocarpa) fruits are rich in anthocyanins, which are vital secondary metabolites that possess antioxidative properties. The aim of this study was to isolate and purify the anthocyanins from black chokeberry by simulated moving bed (SMB) chromatography, and to investigate the neuroprotective effect of $\mathrm{SMB}$ purified anthocyanin against $\mathrm{A} \beta$-induced memory damage in rats. The parameters of the SMB process were studied and optimized. Anthocyanin extracts were identified by HPLC and UPLC-QTOF-MS, and antioxidant abilities were evaluated. The $\mathrm{A} \beta$-induced animal model was established by intracerebral ventricle injection in rat brain. Through the SMB purification, anthocyanins were purified to $85 \%$; cyanidin 3-O-galactoside and cyanidin 3-O-arabinoside were identified as the main anthocyanins by UPLC-QTOF-MS. The SMB purified anthocyanins exhibited higher DPPH and ABTS free radical scavenging abilities than the crude anthocyanins extract. Furthermore, rats receiving SMB purified anthocyanins treatment $(50 \mathrm{mg} / \mathrm{kg})$ showed improved spatial memory in a Morris water maze test, as well as protection of the cells in the hippocampus against $A \beta$ toxicity. These results demonstrate that anthocyanins could serve as antioxidant and neuroprotective agents, with potential in the treatment of Alzheimer's disease.
\end{abstract}

Keywords: black chokeberry; anthocyanin; simulated moving bed; antioxidant activity; neuroprotection; Alzheimer's disease

\section{Introduction}

Black chokeberry (Aronia melanocarpa) is used as an ornamental plant and as a food and colorant. It is rich in the secondary metabolites such as anthocyanins and flavonoids which play vital roles in protecting against oxidative stress and biotic stress [1]. The main anthocyanins in the black chokeberry are cyanidin 3-O-galactoside, cyanidin 3-Oarabinoside, cyanidin 3-O-glucoside, and cyanidin 3-O-xyloside. These compounds exhibit many bioactivities such as antioxidant, antiproliferative, antimicrobial, anti-inflammation, and modulate hepatic lipid metabolism activities [1-4]. Meanwhile, anthocyanins have been shown to prevent and remedy diseases such as cardiovascular disease, liver failure, obesity, and diabetes [5]. Moreover, anthocyanins can cross the blood-brain barrier (BBB) and delay aging-related degenerative diseases [6-8]. However, the stability of anthocyanins is influenced by many factors such as structure, the presence of solvents, $\mathrm{pH}$, temperature, 
oxygen, and enzymes and other concomitant substances; as such it is still impossible to isolate and purify monomeric anthocyanin from complex natural compounds [9].

Neurodegenerative disorders are becoming more and more prevalent, leading to living and economic burdens on the family members of affected individuals. Alzheimer's disease (AD), one of the most common causes of dementia, is associated with many risk factors including alcohol use, smoking, hypertension, exposure to metals, and oxidative stress [10]. A hallmark of AD is the accumulation of insoluble forms of amyloid- $\beta$ (A $\beta$ ) in the plaques in extracellular spaces and in the walls of blood vessels [10]. Although the pathogenesis of $\mathrm{AD}$ is not fully understood, a great deal of research has supported the hypothesis that reactive oxygen species (ROS) impair antioxidant defense systems and induce neuron apoptosis [11]. Many secondary metabolites, like phenolic compounds from Ginkgo biloba, green tea, curcumin, grape, and blueberry, have been reported to protect neuronal cells against oxidative stress in in vivo and in vitro models [12-16].

Simulated moving bed (SMB) chromatography is a continuous countercurrent process which has been used in the separation stage for the large-scale production of compounds such as glucose and fructose [17] and chiral drugs. SMB chromatography is characterized by the separation of a few grams of thermally unstable compounds [18] and chiral drugs, which differ little in terms of their physicochemical properties [19]. Compared to semipreparative liquid chromatography, SMB requires less adsorbent and solvent, and target compounds may be derived with low loss $[17,20]$. Although SMB is suitable for the isolation of unstable secondary metabolites like phenolic compounds, further studies on its use are necessary. To the best of our knowledge, this study is the first to isolate and purify anthocyanins by SMB chromatography and study both their protective effects against oxidative stress and their neuroprotective ability against $A \beta$-induced damage in rats.

The specific aims of this study are to develop a new, highly-efficient method to isolate and purify the anthocyanins from black chokeberry, and to investigate the antioxidant activity of the SMB purified anthocyanin extract. An A $\beta$-induced damage animal model was used to test the effects of SMB-purified anthocyanins on spatial learning and memory ability, as well as nerve cell viability in vivo.

\section{Materials and Methods}

\subsection{Chemicals}

Cyanidin 3-O-glucoside standard (99\%) was purchased from Sichuan Weikeqi Biological Technology CO., LTD (Chengdu, Sichuan, China) and Cyanidin 3-O-galactoside (Cyn-3-gal, 95\%) was obtained from HaoChen Ecological Agriculture Development CO., LTD (Shanghai, China). The A $\beta_{1-40}$ peptides were purchased from Beijing Biosynthesis Biotechnology CO., LTD (Beijing, China). 1,1-diphenyl-2-picrylhydrazyl (DPPH) and 2,2'-azino-bis(3-ethylbenzothiazoline-6-sulfonic acid) diammonium salt (ABTS), 6-hydroxy2,5,7,8-tetramethylchroman-2-carboxylic acid (Trolox) were purchased from TCI (Shanghai) Development CO., LTD (Shanghai, China). All other organic solvents were of analytical grade.

\subsection{Plants Material}

Black chokeberry fruits were harvested at the full maturity stage in September 2017 from black chokeberry demonstration planting base in Wafangdian City $\left(39^{\circ} 49^{\prime} 21^{\prime \prime} \mathrm{N}\right.$, $121^{\circ} 54^{\prime} 32^{\prime \prime} \mathrm{E}$, Dalian, Liaoning, China). Fruits were transported at $4{ }^{\circ} \mathrm{C}$ and stored at $-40{ }^{\circ} \mathrm{C}$ for a maximum of 6 months.

\subsection{Extraction of Anthocyanins}

The black chokeberry fruits were crushed and homogenized using a stainless steel blender, and then extracted twice with $65 \%$ ethanol in a 1:8 (w/v) ratio with $1 \%$ acetic acid in an ultrasonic wave bath for $10 \mathrm{~min}$. Then, the supernatants were collected after centrifugation at $6485 \times g(10,000 \mathrm{rpm}$ in an Anke GL-20G-II centrifuge, Shanghai Anke company, Ltd., Shanghai, China) at $25{ }^{\circ} \mathrm{C}$ for $15 \mathrm{~min}$. The supernatants were evaporated until ap- 
proximately a $90 \%$ volume was achieved, and then loaded onto a column $(2.6 \times 60 \mathrm{~cm})$ containing $100 \mathrm{~g}$ of the Amberlite ${ }^{\circledR}$ XAD-7HP macroporous resin (Sigma Aldrich Co., St. Louis, MO, USA). The column was washed with deionized water, and then eluted with $1 \%$ acetic acid in $35 \%$ ethanol at a flow rate of $1 \mathrm{~mL} / \mathrm{min}$. Then, the crude extract solutions were evaporated, lyophilized, and stored at $-40^{\circ} \mathrm{C}$.

\subsection{Purification of Anthocyanins by $S M B$}

Simulated moving bed chromatography (HYSMB6-500) was provided by Beijing Xiang Yue Huang Yu Technology Development Co., Ltd. (Beijing, China). The mathematical principle model is based on a rigorous mathematical first-principles model and the accurate dynamic models of multicolumn continuous chromatographic processes [18]. The anthocyanin extract solutions were homogenized by ultrasound and dissolved in $25 \%$ ethanol with $1 \%$ acetic acid at a concentration of $50 \mathrm{~g} / \mathrm{L}$ through a $0.45 \mu \mathrm{m}$ membrane to form the raw material solution used as the first SMB feed. In this SMB system (Figure 1), a four-zone working model was set up including an eluting zone (zone I), a refining (zone II) and an adsorbing zone (zone III), and a washing zone (zone IV) with two columns per zone. The SMB was equipped with eight columns $(10 \mathrm{~mm} \times 150 \mathrm{~mm})$ with C18 $(30 \mu \mathrm{m})$ filler configured as $2 / 2 / 2 / 2$. The fluid was $25 \%$ ethanol with $1 \%$ acetic acid. Pump F and $\mathrm{D}$ pumped into the feeding and desorbent for eluting, and pump $\mathrm{E}$ and $\mathrm{R}$ pumped out the extract and raffinate. $Q_{E}, Q_{R}, Q_{D}$, and $Q_{F}$ are the flow rates of extract, raffinate, desorbent and feed, respectively. The SMB purified anthocyanin extract (SMB ACN) solutions were dried under vacuum in a rotary evaporator and lyophilized into powder, and then stored at $-40{ }^{\circ} \mathrm{C}$. HPLC-PDA was used to monitor of the extract and raffinate during the SMB process, and the purity was calculated by the area of the corresponding anthocyanin peak to the total peak area at $278 \mathrm{~nm}$.

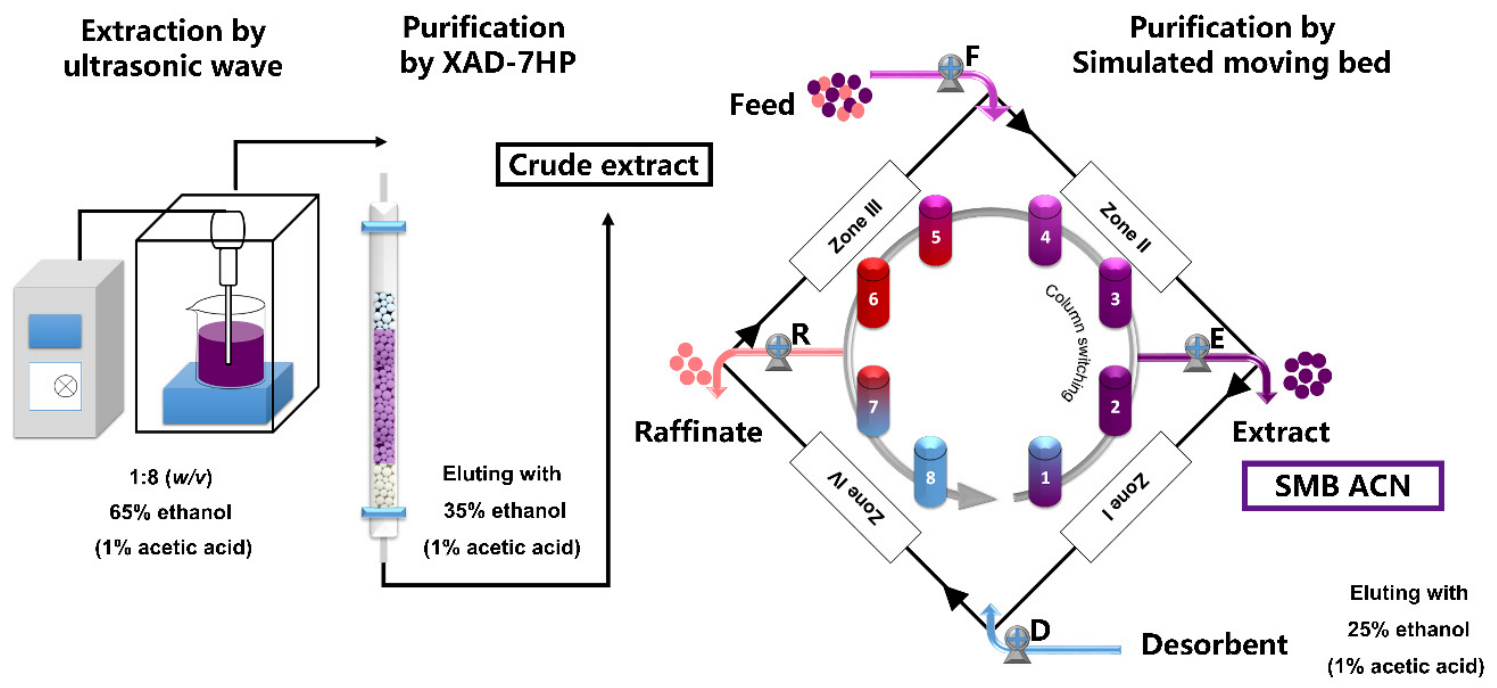

Figure 1. Schematic illustration of the isolation and purification of anthocyanins from black chokeberry fruits. The anthocyanins were isolated by an ultrasonic extraction method and the crude extract was prepared by XAD-7HP macroporous resins as the feed of simulated moving bed chromatography (SMB). The separation zones of SMB were zones II and III, and the solid phase was regenerated in zones I and IV with two columns per zone. The liquid phase (black) consisted of feed and desorbent for inlets, and extract and raffinate for outlets by pump E and pump R. The liquid phase was continuously fed the crude extract by pump F and eluted with ethanol by pump D. Then, SMB ACN was collected from the extract. The columns switched counter-clockwise (gray) at the switching time intervals. 


\subsection{HPLC-PDA and UPLC-QTOF-MS Analysis}

The anthocyanins extract powders were dissolved in water with $10 \%$ acetic acid and $1 \%$ phosphoric acid. Then, all samples were centrifuged at $9339 \times g(12,000 \mathrm{rpm})$ at $4{ }^{\circ} \mathrm{C}$ for $10 \mathrm{~min}$, and the supernatants were used for HPLC-PDA and UPLC-QTOF-MS analysis.

The HPLC system consisted of a pump (LC-20 AT) and a photodiode array (PDA) detector (SPD-10A) (Shimadzu Corp., Tokyo, Japan). The analytical column was the Intertsil ODS-SP C18 column $(4.6 \times 250$ mm, $5 \mu \mathrm{m}$, GL Sciences Inc., Tokyo, Japan). Mobile phase A was $10 \%$ acetic acid and $1 \%$ phosphoric acid in water, and mobile phase B was $100 \%$ acetonitrile. Elution was performed at a flow rate of $1.0 \mathrm{~mL} / \mathrm{min}$, and the solvent gradient was as follows: $0-8 \mathrm{~min}, 10 \% \mathrm{~B} ; 8-12 \mathrm{~min}$, B from $10 \%$ to $40 \% ; 12-15 \mathrm{~min}, 40 \% \mathrm{~B}$; $15-25 \mathrm{~min}$, from $40 \%$ to $10 \% \mathrm{~B}$. The injection volume was $20 \mu \mathrm{L}$ and the column was thermostated at $25^{\circ} \mathrm{C}$. The analysis wavelengths were $278 \mathrm{~nm}$ for monitoring the SMB process and $520 \mathrm{~nm}$ for quantifying the anthocyanins [21]. The UV-VIS spectra were scanned from 220 to $800 \mathrm{~nm}$. Cyanidin 3-O-galactoside were quantified using cyanidin 3 -O-galactoside $\left(y=18,674 x+11,660, \mathrm{R}^{2}=0.9994\right)$ and cyanidin 3-O-glucoside, cyanidin 3-O-arabinoside and cyanidin 3-O-xyloside were quantified using cyanidin 3-O-glucoside $\left(y=25,228 x+10,850, R^{2}=0.9990\right)$. The purity was determined by the area normalization method of these four anthocyanins on HPLC-PDA at $278 \mathrm{~nm}$. The anthocyanin contents were determined by comparing retention times and absorption spectra, and further confirmed by UPLC-QTOF-MS.

A Waters ACQUITY UPLC system coupled with a Xevo G2QTOF mass spectrometer (Waters Corporation, Milford, MA, USA) was used for anthocyanins confirmation as previously described [22]. The separation of anthocyanins was conducted on a HSS T3 column $(2.1 \mathrm{~mm} \times 100 \mathrm{~mm}, 1.8 \mu \mathrm{m})$. The mobile phase was $(\mathrm{A})$ water $+0.2 \%$ formic acid and (B) acetonitrile $+0.2 \%$ formic acid: 0 to $1 \mathrm{~min}, 5 \% \mathrm{~B} ; 1$ to $2 \mathrm{~min}, 5 \%$ to $40 \% \mathrm{~B} ; 4$ to $8 \mathrm{~min}$, $95 \% \mathrm{~B} ; 8.1$ to $10 \mathrm{~min}, 5 \% \mathrm{~B}$. The injection volume was $2 \mu \mathrm{L}$ and the flow rate was $0.3 \mathrm{~mL} / \mathrm{min}$. The ESI parameters were set as follows: negative mode, capillary voltage $2.2 \mathrm{kV}$, sampling cone voltage $30 \mathrm{~V}$, extraction cone voltage $4 \mathrm{~V}$, source temperature $100{ }^{\circ} \mathrm{C}$, desolvation gas (nitrogen) temperature $400{ }^{\circ} \mathrm{C}$, cone gas flow rate $20 \mathrm{~L} / \mathrm{h}$, desolvation gas (nitrogen) flow rate $800 \mathrm{~L} / \mathrm{h}$, collision energies $6 \mathrm{eV}$ and mass range from 50 to $1200 \mathrm{Da}$. The anthocyanin structure was determined according to its fragmentation pattern of deprotonated and product ions.

\subsection{DPPH Free Radical Scavenging Assay}

The ROS scavenging capacity was determined by the DPPH free radical scavenging assay as previously reported [23]. The assay was performed in a 96-well plate using serial dilutions of $5 \mu \mathrm{L}$ aliquots of each Cyn-3-gal (14 to $449 \mathrm{mg} / \mathrm{L}$ ), anthocyanins crude extract ( 2.5 to $80.0 \mathrm{mg} / \mathrm{L})$ and SMB ACN (0.03 to $1.0 \mathrm{~g} / \mathrm{L})$. DPPH solution $(200 \mu \mathrm{L}, 0.06 \mathrm{mM})$ was added to each well, and the plate was incubated at room temperature in the dark for $30 \mathrm{~min}$. The absorbance was determined at $520 \mathrm{~nm}$ using a Thermo Multiskan MK3 Automated Microplate Reader (Thermo Fisher Scientific, Waltham, MA, USA). The EC $_{50}$ value was calculated using a calibration curve of Trolox ( 25 to $250 \mathrm{mg} / \mathrm{L}$ ) by SPSS.

\subsection{ABTS Free Radical Scavenging Assay}

The ABTS•+ scavenging capacity assay was determined as previously described with some modifications [24]. The ABTS $\bullet+$ solution was produced by reacting aqueous ABTS solution $(7 \mathrm{mM})$ with potassium persulfate $(2.45 \mathrm{mM})$. Diluted ABTS $\bullet+$ solution with an absorbance of $0.70 \pm 0.02$ at $734 \mathrm{~nm}$ was employed in the analysis. The reactions were performed by adding $4 \mathrm{~mL}$ of ABTS $\bullet+$ solution and $0.4 \mathrm{~mL}$ of each Cyn-3-gal (14 to $449 \mathrm{mg} / \mathrm{L})$, anthocyanins crude extract (2.5 to $40.0 \mathrm{~g} / \mathrm{L})$ and SMB ACN $(0.03$ to $1.0 \mathrm{~g} / \mathrm{L})$. After 6 min of incubation at room temperature, absorbance values were measured on a spectrophotometer at $734 \mathrm{~nm}$. The $\mathrm{EC}_{50}$ value of ABTS $\bullet+$ scavenging capacity was calculated using a Trolox calibration curve ( 25 to $250 \mathrm{mg} / \mathrm{L}$ ). 


\subsection{Experimental Animals}

Thirty-six Sprague-Dawley (SD) rats (male, weighing $250 \pm 20 \mathrm{~g}$, specific pathogenfree) were purchased by Beijing HFK Bioscience Co. Ltd. (Beijing, China). The rats were kept at the animal facility with free access to standard chow and water at $22 \pm 2{ }^{\circ} \mathrm{C}$, the relative humidity of $45 \pm 15 \%$ and $12 \mathrm{~h}$ light/dark cycle. All animals had adapted for one week before experiments. SD rats were randomly divided into 3 groups $(n=12)$ of sham, $A \beta, A \beta+$ SMB ACN. The group of $A \beta$ and $A \beta+$ SMB ACN were treated by intracerebral ventricle injection of $A \beta_{1-40}$, and then $A \beta+S M B A C N$ group were administered by intragastric administration with anthocyanins $(50 \mathrm{mg} / \mathrm{kg}$ anthocyanins $85 \%$ ) for one month. The experiment was approved by the Animal Ethical and Welfare Committee (No. IRMDWLL-2017095).

\subsection{Preparation of $A \beta$-Induced Damage Rat Model}

The $A \beta_{1-40}$ oligomers were prepared according to a previously reported method [25]. Briefly, $A \beta_{1-40}$ peptide was prepared as a stock solution at a concentration of $1 \mathrm{mg} / \mathrm{mL}$ in sterile sodium chorionic solution, followed by aggregation via incubation at $37^{\circ} \mathrm{C}$ for 4 days.

Rats were anesthetized with $10 \%$ chloral hydrate $(0.3 \mathrm{~mL} / 100$ g i.p. $)$ and the head was symmetrically held in the stereotaxic apparatus (ZS-FD, ZS Dichuang, Beijing, China). The scalp skin was clean shaved and the skull was exposed. One hole was drilled on the skull at coordinates of $-0.8 \mathrm{~mm}$ posterior to the bregma and $1.4 \mathrm{~mm}$ lateral according to the atlas of Paxinos and Watson [26,27]. The needle was injected $4 \mathrm{~mm}$ deep into the ventricle with a speed of $1 \mu \mathrm{L} / \mathrm{min}$ for $5 \mu \mathrm{L}$ and left in place for 5 min after the injection of $A \beta_{1-40}$. The sham rats were injected with $0.9 \%$ saline. The surgical wound was sutured and the animals were returned to their cages with free access to food and water, and allowed to recover for 1 day [28].

\subsection{Morris Water Maze}

After one month of intragastric administration with anthocyanins, the Morris water maze (MWM) test was performed as previously described [29]. The circular pool was $150 \mathrm{~cm}$ in diameter and $60 \mathrm{~cm}$ in depth with an invisible platform $(12 \mathrm{~cm}$ in diameter and $25 \mathrm{~cm}$ in depth). The water in the pool was mixed with black ink. During the experiment, the temperature of the water remained within 22 to $24^{\circ} \mathrm{C}$, and all landmarks around the maze remained the same. The MWM included spatial learning and acquisition trials (hidden platform trials) and a spatial probe trial. Before the spatial acquisition trials, each rat was put into the water to adapt for $2 \mathrm{~min}$ and the platform was visible, located in the middle of the first quadrant for one day. Then, water was added to the pool with the platform $1 \mathrm{~cm}$ below the water surface. During the hidden platform trials, each rat was placed into the water from one of the start positions, facing the wall. The spatial acquisition trials were conducted over four days, with four trials per day. If the animal reached the platform, the timer was stopped. If the animal failed to find the platform within $90 \mathrm{~s}$, the animal was placed on the platform for $15 \mathrm{~s}$ to help it learn the platform's location. The spatial probe test was on day 5 , and then the platform was removed. The rats were released from the third quadrant $\left(180^{\circ}\right.$ from the original platform position) and the time spent crossing the target quadrant and the number of times the region in which the platform was previously located was crossed were recorded over a $90 \mathrm{~s}$ period. After testing, the rats were dried with a towel to keep them warm. The animals were recorded with a video camera, and the data were analyzed using ANY-maze behavioral tracking software (Stoelting Co., Wood Dale, IL, USA)

\subsection{Nissl Staining}

For Nissl staining of the brain, the rats were euthanized by intraperitoneal injection of $0.3 \%$ chloral hydrate, and were transcardially perfused with $100 \mathrm{~mL}$ of saline $(0.9 \% w / v$ $\mathrm{NaCl}$ ). Then, the brains were removed and put in the $15 \mathrm{~mL}$ of $4 \%$ paraformaldehyde. 
Serial coronal hippocampal sections with a thickness of $25 \mu \mathrm{m}$ were cut using a cryostat (Leica Microsystems, Wetzlar, Germany). The sections were washed twice for $5 \mathrm{~min}$ in $0.01 \mathrm{M}$ PBS and incubated in 1\% toluidine blue staining solution for 5-10 min at room temperature. Then, the sections were rinsed in distilled water, soaked in $95 \%$ ethanol for $30 \mathrm{~min}$, and dehydrated in $100 \%$ ethanol. After dehydration, brains were placed in xylene and cover slipped using resin medium. The neurons were quantified using image software (Image J $1.45 \mathrm{~s}$ ), with three sections from three rats for each group.

\subsection{Statistical Analysis}

The data are presented as mean \pm SD in triplicate from at least using GraphPad Prism 7.0 software (GraphPad Software, San Diego, CA, USA). $p<0.05$ was considered statistically significant by LSD test. The results were analyzed by ANOVA and the $\mathrm{EC}_{50}$ value was calculated using the Probit Analysis by SPSS 20.0 software (IBM, Armonk, NY, USA).

\section{Results}

\subsection{Isolation and Purification of Black Chokeberry Anthocyanins by SMB}

Anthocyanins from black chokeberry were extracted and preliminarily purified by the XAD-7HP macroporous resin column. Four-section SMB was employed to separate the anthocyanins crude extract. After approximately 20 switching times, a cyclic steady state was reached. As shown in Table 1, the optimal parameters of flow rate were $\mathrm{Q}_{\mathrm{F}}=0.42 \mathrm{~mL} / \mathrm{min}$, $\mathrm{Q}_{\mathrm{E}}=1.5 \mathrm{~mL} / \mathrm{min}, \mathrm{Q}_{\mathrm{R}}=6.5 \mathrm{~mL} / \mathrm{min}$, and $\mathrm{Q}_{\mathrm{D}}=4.5 \mathrm{~mL} / \mathrm{min}$. The HPLC-PDA chromatograms of feed, raffinate and extract by running $\mathrm{H}$ are presented in Figure 2A-C. Anthocyanins and impurities are separated in column 3 to 6 (Figure 1). The purity of the SMB ACN was estimated to be $68 \%$ to $85 \%$. These results in run A, B and G indicated that a switching time of $141 \mathrm{~s}$ maximally purified the anthocyanins. At a certain switching time, the slight decrease of flow rate in the feed, extract and raffinate zone increased the purity comparing run $\mathrm{B}$ to $\mathrm{C}, \mathrm{E}$ to $\mathrm{G}$ and $\mathrm{D}$ to $\mathrm{H}$. These results confirmed that the performance of SMB was mainly associated with the switching time and flow rate of feed, extract and raffinate [30].

Table 1. Operation parameters and separation performance of the SMB.

\begin{tabular}{|c|c|c|c|c|c|c|}
\hline \multirow{2}{*}{ Run } & \multicolumn{4}{|c|}{ Flow Rates (mL/min) } & \multirow{2}{*}{ Switch Time ts (s) } & \multirow{2}{*}{ Purity (\%) } \\
\hline & $\mathrm{Q}_{\mathrm{E}}$ & $Q_{R}$ & $Q_{D}$ & $\mathbf{Q}_{\mathbf{F}}$ & & \\
\hline $\mathrm{A}$ & 1.5 & 6.5 & 4.5 & 0.45 & 135 & 68.7 \\
\hline B & 1.5 & 6.5 & 4.5 & 0.45 & 140 & 78.2 \\
\hline $\mathrm{C}$ & 1.5 & 6.5 & 4.5 & 0.40 & 140 & 73.1 \\
\hline $\mathrm{D}$ & 1.5 & 8.0 & 4.5 & 0.42 & 141 & 71.4 \\
\hline $\mathrm{E}$ & 3.5 & 6.5 & 4.5 & 0.45 & 141 & 70.3 \\
\hline $\mathrm{F}$ & 1.5 & 6.0 & 4.5 & 0.42 & 141 & 77.5 \\
\hline G & 1.5 & 6.5 & 4.5 & 0.45 & 141 & 78.3 \\
\hline $\mathrm{H}$ & 1.5 & 6.5 & 4.5 & 0.42 & 141 & 85.1 \\
\hline
\end{tabular}

In order to analyze the anthocyanins crude extract and SMB CAN, HPLC-PDA was used to quantify and UPLC-QTOF-MS to identify the anthocyanins. An HPLC chromatogram of the crude extract showed four peaks (Figure $3 \mathrm{~A}$ ). The $\lambda_{\max }$ value of UV-VIS spectra was $516 \mathrm{~nm}$ for cyanidin. The SMB ACN mainly showed two peaks by HPLC-PDA (Figure 3B) which were identified by UPLC-QTOF-MS (Figure 3C,D). Peak 1 of cyanidin 3-O-galactoside ( $\mathrm{m} / \mathrm{z} 449.1089 / 287.0560)$ appeared as a principal peak, and peak 2 was identified as a cyanidin 3-O-arabinoside by detection of the respective parent and product ion pairs ( $\mathrm{m} / \mathrm{z} 419.0975 / 287.0557)$. The four compounds of crude extract were cyanidin 3 -O-galactoside $(72.9 \%$ of total anthocyanins), cyanidin $3-O$-glucoside $(2.7 \%)$, cyanidin 3-O-arabinoside $(21.0 \%)$, and cyanidin 3-O-xyloside (3.4\%) (Table 2$)$, which is in accordance with the Oszmianski's results [31]. The mass of total anthocyanins in the crude extract was 
$2.29 \pm 0.19 \mathrm{~g} / 100 \mathrm{~g}$ of dry extract, and the mass of total anthocyanins in the SMB ACN was $61.02 \pm 6.46 \mathrm{~g} / 100 \mathrm{~g}$ of the fraction rich in dry anthocyanins.
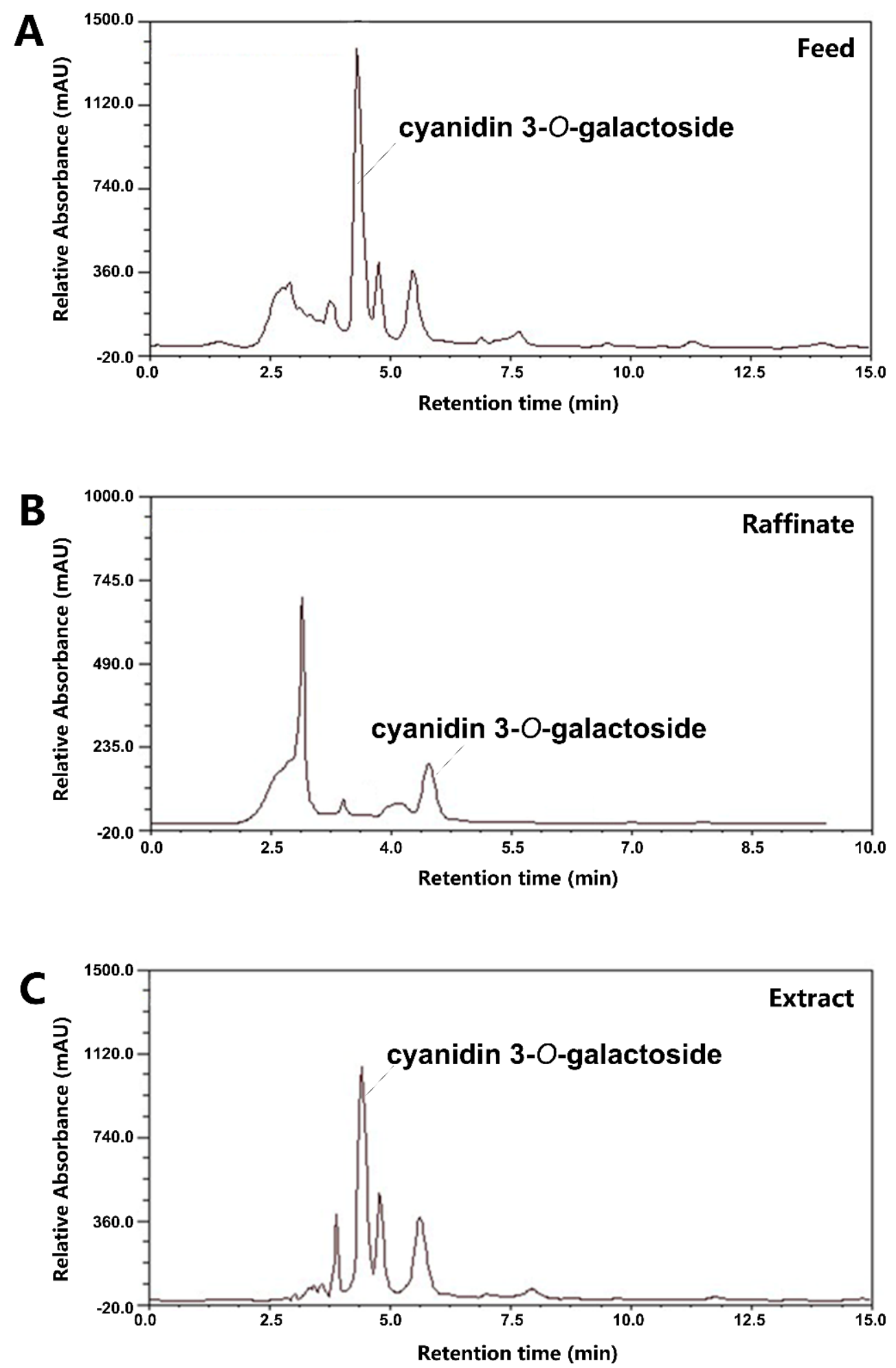

Figure 2. HPLC-PDA chromatogram of the feed (A), the raffinate $(\mathbf{B})$, and the extract $(\mathbf{C})$ during the $\mathrm{SMB}$ process monitored at $278 \mathrm{~nm}$. 

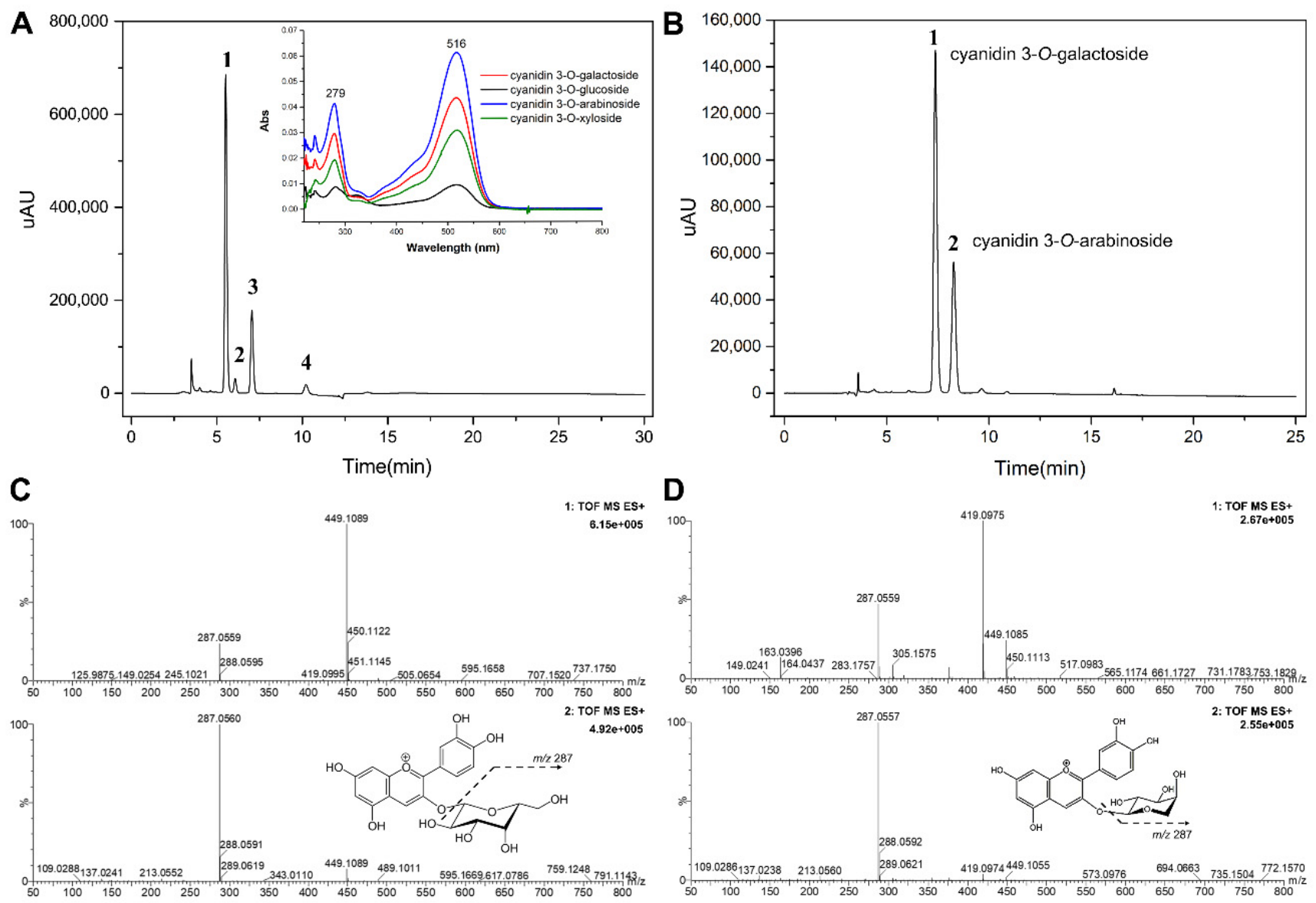

Figure 3. (A) HPLC-PDA chromatogram and UV-VIS scanning spectra of the black chokeberry anthocyanins crude extract at $520 \mathrm{~nm}$ and peak identities (1: cyanidin 3-O-galactoside; 2: cyanidin 3-O-glucoside; 3: cyanidin 3-O-arabinoside; 4: cyanidin 3-O-xyloside); (B) HPLC-PDA chromatogram of the SMB ACN at $520 \mathrm{~nm}$ and peak identities (1: cyanidin 3-O-galactoside; 2: cyanidin 3-O-arabinoside); The UPLC-QTOF-MS mass spectra and structure of cyanidin 3-O-galactoside (C) and cyanidin 3-O-arabinoside (D) of the SMB ACN peak 1 and peak 2.

Table 2. HPLC quantification and UPLC-QTOF-MS identification of anthocyanins in Black chokeberry crude extract and SMB ACN.

\begin{tabular}{|c|c|c|c|c|c|c|}
\hline Peak & RT HPLC & Compound & Molecular Formula & ESI(+)MS/MS2 & Crude Extract & SMB ACN \\
\hline & $(\min )$ & & & $(\mathrm{m} / \mathrm{z})$ & (mg/100 g FW) & (mg/g DW) \\
\hline 1 & 4.809 & cyanidin 3-O-galactoside & $\mathrm{C}_{21} \mathrm{H}_{21} \mathrm{O}_{11}$ & $\begin{array}{c}449.1089([\mathrm{M}]+) \\
287.0560([\mathrm{M}-\mathrm{gal}]+)\end{array}$ & $500.4 \pm 38.7$ & $449.1 \pm 30.8$ \\
\hline 2 & 5.169 & cyanidin 3-O-glucoside & $\mathrm{C}_{21} \mathrm{H}_{21} \mathrm{O}_{11}$ & $\begin{array}{c}449.1088([\mathrm{M}]+) \\
287.0558([\mathrm{M}-\mathrm{glu}]+)\end{array}$ & $18.6 \pm 1.8$ & ND \\
\hline 3 & 5.923 & cyanidin 3-O-arabinoside & $\mathrm{C}_{20} \mathrm{H}_{19} \mathrm{O}_{10}$ & $\begin{array}{c}419.0975([\mathrm{M}]+) \\
287.0557([\mathrm{M}-\mathrm{arab}]+)\end{array}$ & $144.2 \pm 14.0$ & $161.2 \pm 33.8$ \\
\hline 4 & 8.219 & cyanidin 3-O-xyloside & $\mathrm{C}_{20} \mathrm{H}_{19} \mathrm{O}_{10}$ & $\begin{array}{c}419.0975([\mathrm{M}]+) \\
287.0556([\mathrm{M}-x y l]+)\end{array}$ & $23.4 \pm 1.8$ & ND \\
\hline
\end{tabular}

Data presented in fresh fruit weight (FW) and dried extract weight (DW). ND: not detected.

The SMB continuously purified the anthocyanins, and efficiently enriched the two main anthocyanins and eliminated the impurity. A similar observation was made by Wang, who reported the use of a two-step simulated moving bed chromatographic process to purify the EGCG from tea polyphenol [32]. It was suggested that the SMB was a highly efficient way to separate and purify the secondary metabolites from a natural extract. Due to the instability of secondary metabolites like anthocyanins and the complexity of the plant phenols, the extraction process of monomer was extremely difficult. Therefore, 
these results indicated that SMB may be a feasible and effective strategy to isolate and purify the anthocyanins from black chokeberry.

\subsection{The Free Radical Scavenging Abilities of Black Chokeberry Anthocyanin Extracts}

To evaluate the SMB purification process, we tested the DPPH and ABTS free radical scavenging abilities of the black chokeberry anthocyanins crude extract and the SMB purified anthocyanin extract. As shown in Figure 4, the $\mathrm{EC}_{50}$ values of anthocyanins crude extract were significantly higher than Trolox, Cyn-3-gal and SMB ACN of the free radical scavenging abilities $(p<0.001)$. The DPPH $\bullet$ and ABTS $\bullet+$ scavenging of crude extract $(54.18 \pm 19.59 \mathrm{~g} / \mathrm{L}$ and $4.21 \pm 1.50 \mathrm{~g} / \mathrm{L})$ were 65 - and 30-fold more than SMB ACN $(0.83 \pm 0.20 \mathrm{~g} / \mathrm{L}$ and $0.14 \pm 0.06 \mathrm{~g} / \mathrm{L})$. The lower $\mathrm{EC}_{50}$ of SMB ACN indicated a higher antioxidant activity. Moreover, the ABTS radical scavenging ability of SMB ACN was similar to Cyn-3-gal and Trolox $(p>0.05)$. These results suggest that the SMB process increased the free radical scavenging ability of the anthocyanin extract.

A

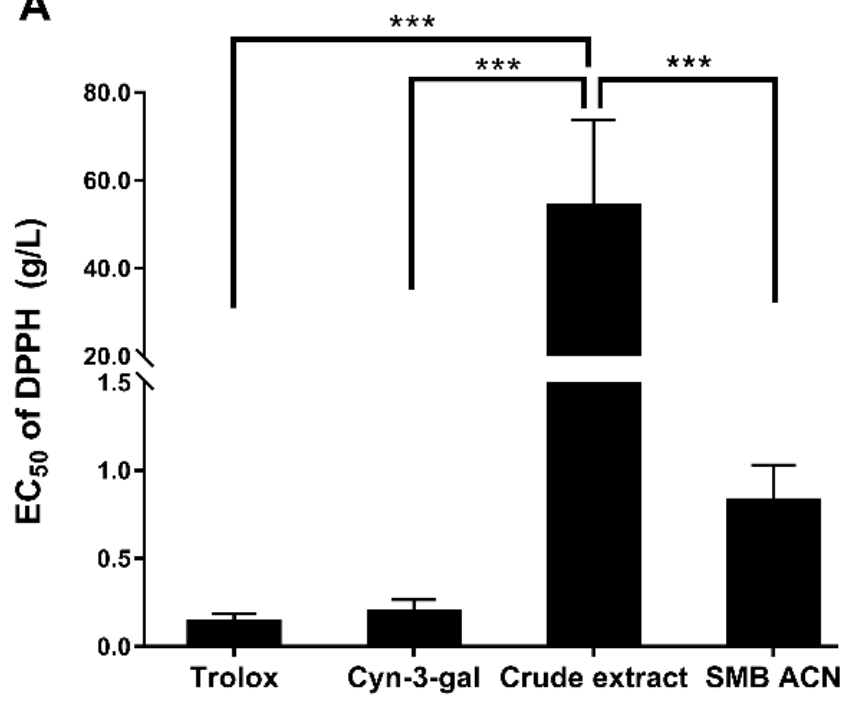

B

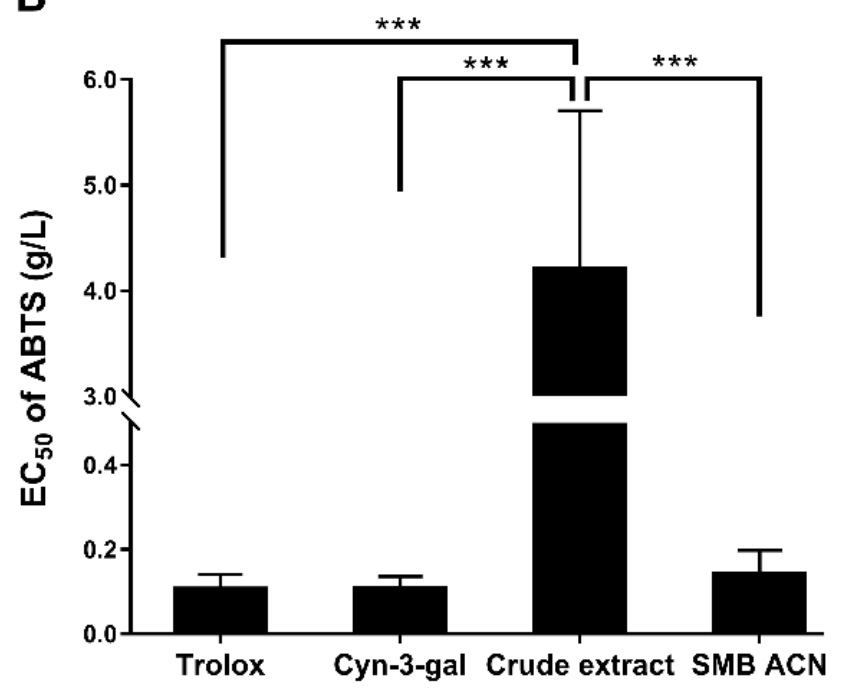

Figure 4. (A) DPPH free radical scavenging activity of Trolox, cyanidin 3-O-galactoside (Cyn-3-gal), anthocyanins crude extract and SMB ACN; (B) ABTS free radical scavenging activity of Trolox, Cyn-3-gal, anthocyanins crude extract and $\mathrm{SMB} A C N$. Date represent mean $\pm \mathrm{SD}(n=6)$ were expressed by the $\mathrm{EC}_{50}$ inhibition of the free radical scavenging and the *** $p<0.001$ by LSD test of one-way ANOVA.

The results showed that the ABTS free radical scavenging abilities of anthocyanins extracts were higher than those of DPPH; notably, cyanidin 3-O-galactoside had a high free radical scavenging ability due to its hydroxyl group. It may be that the anthocyanin extracts neutralized the DPPH free radicals mainly by hydrogen atom transfer and the ABTS $\bullet+$ by a fast, non-selective electron transferring process. Therefore, the application of the SMB chromatography purified and enrichened the anthocyanins and improved the antioxidant ability than crude extract. Deneva et al. found that anthocyanins were the second biggest contributor to antioxidant activity after the proanthocyanidins among black chokeberry polyphenols [3]. Moreover, cyanidin-3-O-glucoside could regulate cellular antioxidant defense induced by A $\beta_{1-40}$ in SH-SY5Y cells through the Nrf2 signaling pathway [33]. The purified anthocyanins may scavenge the free radicals and inhibit the $A \beta_{1-40}$ neurotoxicity. Therefore, the next section of this study is concerned with SMB ACN, investigating its neuroprotective activity against amyloid- $\beta$ induced in rats.

\subsection{The Protection of Anthocyanins on Memory Impairment in A $\beta$-Induced Toxicity Rats}

The Morris water maze (MWM) is a method to assess spatial learning and memory in rats. The escape latency time of all groups had a decreasing tendency over four days 
(Figure 5A). The results showed that the SMB ACN treatment group presented a less steep learning curve between days 1 and 2, which might indicate that anthocyanins had increased the short-term memory. The sham group showed a more stable spatial learning rate than the $A \beta+S M B A C N$ group. In the probe trial, the time spent in the targeted quadrant of the sham group was shorter compared to the $A \beta$ group impaired by $A \beta_{1-40}$, which indicated that the $A \beta$ group showed lower long-tern learning and memory ability (Figure 5B). The rats given the anthocyanins extract achieved more platform crossings in comparison with the $\mathrm{A} \beta$-induced group, and showed a nonsignificant difference with the sham group (Figure 5C). The results indicated that anthocyanin group had a significantly higher spatial learning ability than the $\mathrm{A} \beta$-induced group $(p<0.05)$. Interestingly, the sham group achieved higher scores than the $A \beta$ group, although this was not significant, and may have been due to intervariability among the rats. As shown in Figure 5D, the A $\beta+$ SMB $A C N$ group allayed behavioral deficits compared to the $A \beta$ group regarding the swim path. The behavioral test results showed that the purified anthocyanins extract alleviated the damage induced by amyloid- $\beta$ to spatial learning and memory.

A

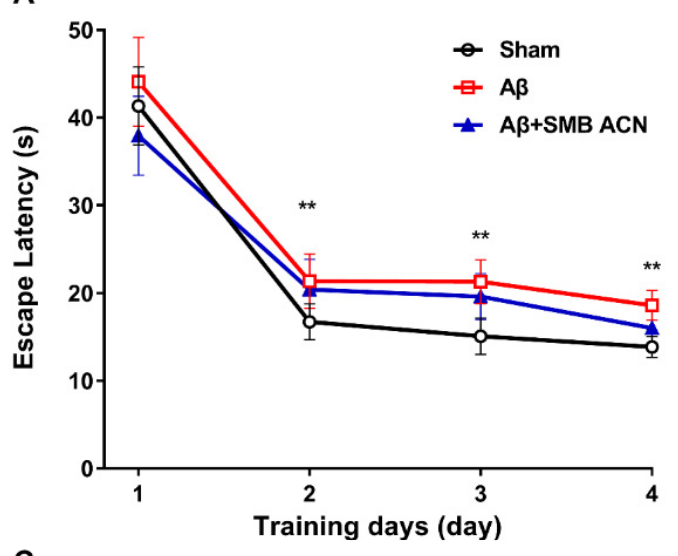

C

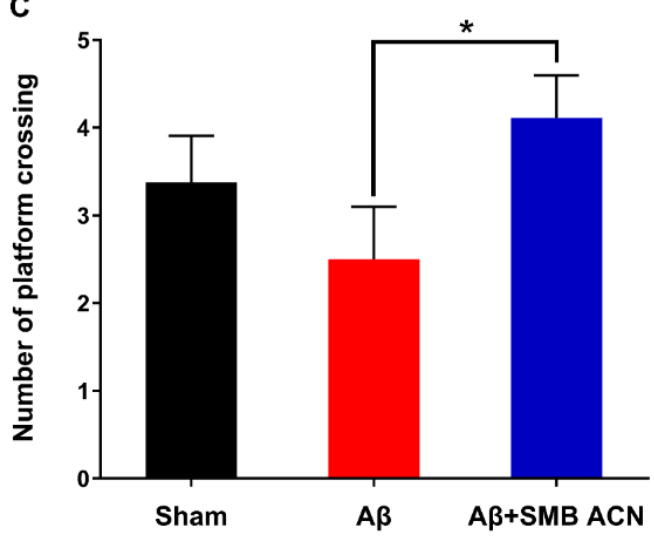

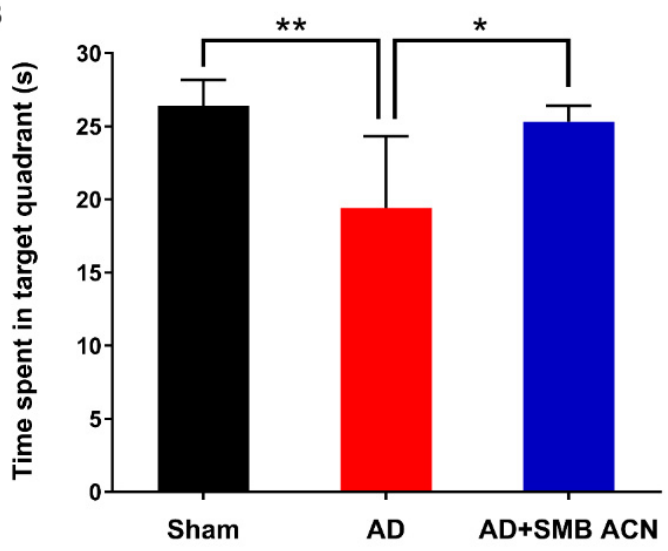

D

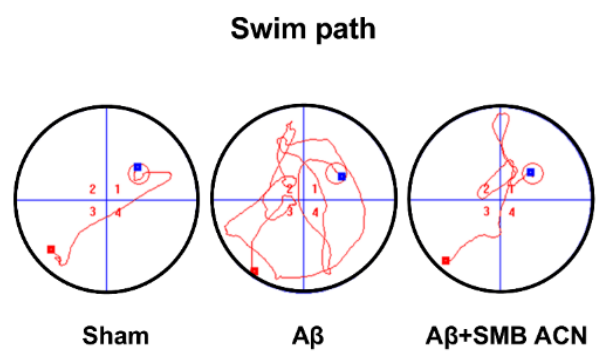

Figure 5. SMB ACN treatment alleviated $A \beta$ induced spatial learning deficits. (A) The escape latency during the spatial acquisition trials of Sham, $\mathrm{A} \beta$ and $\mathrm{A} \beta+\mathrm{SMB} \mathrm{ACN}$ group; (B) The time spent in the target quadrant of the spatial probe test; (C) The number of platform crossing of the spatial probe test; (D) The swim path of the spatial acquisition trial. The results were expressed by mean $\pm \mathrm{SD}(n=12)$ and the $* p<0.05,{ }^{* *} p<0.01$ by LSD test of one-way ANOVA.

Previous reports have shown that anthocyanin galactosides are better maintained in the intestines compared to glucosides, while arabinosides or xylosides showed negligible losses [34]. Notably, cyanidin 3-O-galactoside and cyanidin 3-O-arabinoside crossed the blood-brain barrier into the cortex and hippocampus [16]. Moreover, the neuroprotective ability of black chokeberry has been reported; additionally, anthocyanins have been associated with antiaging [7]. Anthocyanins were shown to regulate the cell cycle and senescence by regulating the expression of the DNA damage signaling pathway and an- 
tioxidant enzymes in neuronal cells $[7,11]$. Our results support the hypothesis that the administration of SMB ACN ameliorates learning and memory impairment by $\mathrm{A} \beta$-induced severe behavioral dysfunction.

\subsection{The Neuroprotective Effect of SMB Anthocyanins Extract in Rat Brain}

To further elucidate the neuroprotective effect of anthocyanins, we examined the hippocampus. The Nissl staining stains the nuclei of all cells, as well as clumps of material surrounding the nuclei of neurons. Neuronal loss in the brain, especially in the CA1 region of the hippocampus [35] and basal forebrain, is one of the most important pathological hallmarks of Alzheimer's disease [36]. The pyramidal cells in the CA1 and CA3 regions of the hippocampus in the brain modulates memory and emotions [37].

The results showed that the survival of neuron cells in the CA1 and CA3 of the sham group was greater than in the $A \beta_{1-40}$ treated group $(p<0.05)$ (Figure 6). The neurons in the $A \beta$ group were disorganized and deformed with deep stain nuclei due to the neurotoxicity of $A \beta$. The results of the sham group indicated that the injection into the ventricle of the brain did not damage the hippocampus region. The neurons and kernel in the visual field of the $A \beta+$ SMB ACN group were clearer and more intact than the $A \beta$ group, and the numbers of pyramidal cells in the CA1 and CA3 were significantly increased $(p<0.05)$. The neurons in the $A \beta_{1-40}$ treated group were damaged with extensive degenerative changes including sparse cell arrangements, swollen cell bodies, loss of integrity, shrunken cytoplasm and oval or triangular nucleus $[38,39]$. These results confirmed that $\mathrm{A} \beta_{1-40}$ can induce cellular loss and disorganization of the pyramidal cells, and indicated that the treatment of anthocyanins can reverse these changes to alleviate the loss of memory.
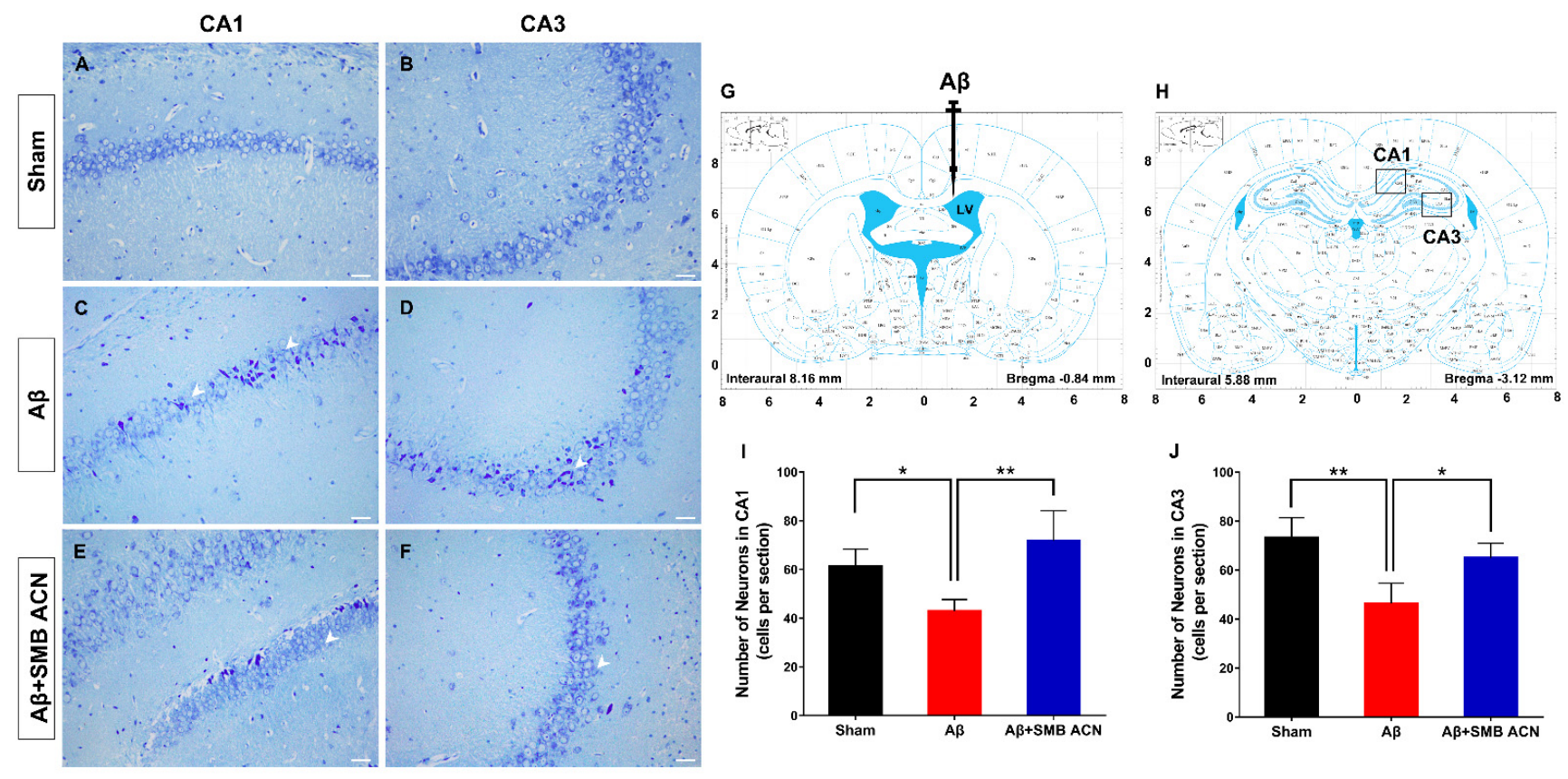

Figure 6. Representative images of Nissl staining in the CA1 and CA3 regions of the hippocampus of sham group (A,B), $A \beta$ group $(\mathbf{C}, \mathbf{D})$, and $A \beta+\operatorname{SMB} A C N$ group $(\mathbf{E}, \mathbf{F}) .(\mathbf{G})$ The site of intracerebral ventricle injection in rat brain (Black) and the sites of the section in CA1 and CA3 region of the hippocampus $(\mathbf{H})$. The number indicated the surviving neurons in CA1 (I) and CA3 (J), ${ }^{*} p<0.05$ and ${ }^{* *} p<0.01$. Stained sections of each group were viewed at $200 \times$ magnification. Scale bar $=100 \mu \mathrm{m}$.

This research shows that purified anthocyanins by SMB exhibit excellent antioxidative activity against oxidative stress and prevent amyloid- $\beta$ induced neurotoxicity in the brain. However, we still need to investigate the mechanism of antioxidative ability in vivo and the bioavailability of anthocyanins and their metabolites to determine the efficacy of such a 
treatment. The cell signaling pathway of anthocyanins regulating the cellular antioxidant system and alleviating the A $\beta$ neurotoxicity still needs to be examined. We used UPLCQTOF-MS to analyze a black chokeberry anthocyanins crude extract and found many secondary metabolites, such as polyphenols, that have antioxidant activities. In a future study, the neuroprotective ability of black chokeberry may found to be associated with the ability of these secondary metabolite compounds to protect neuronal cells against oxidative stress.

\section{Conclusions}

In this study, the anthocyanins from black chokeberry (Aronia melanocarpa) were isolated and purified by XAD-7HP macroporous resin and SMB chromatography, yielding a purity of $85 \%$. The main purified anthocyanins (cyanidin 3-O-galactoside and cyanidin 3-O-arabinoside) had a strong antioxidant ability and ameliorated learning and memory impairment among $\mathrm{A} \beta$-induced neurotoxicity rats. Taken together, these results demonstrate that simulated moving bed chromatography could be a feasible and effective strategy to separate highly bioactive anthocyanins from black chokeberry. The present study was an attempt to establish a method to isolate secondary metabolites from natural plants to exploit their neuroprotective abilities.

Author Contributions: Conceptualization, L.M. and J.L.; methodology, H.W., H.C., and H.T.; software, H.W. and X.Z.; validation, X.Z. and C.R.; investigation, H.W., H.C., and H.T.; data curation, L.M. and J.L.; writing—original draft preparation, H.W. and X.Z.; writing-review and editing, H.W., H.C., C.R. and J.L.; visualization, H.W.; supervision, L.M. and J.L.; project administration, J.L. All authors have read and agreed to the published version of the manuscript.

Funding: This work was funded by the National Natural Science Foundation of China, grant number 31571840 .

Institutional Review Board Statement: The study was approved and conducted according to the Animal Ethical and Welfare Committee (Approval No. IRM-DWLL-2017095).

Informed Consent Statement: Not applicable.

Data Availability Statement: The data are not publicly available because the research is still ongoing.

Conflicts of Interest: The authors declare no conflict of interest.

\section{References}

1. Kim, B.; Ku, C.S.; Pham, T.X.; Park, Y.; Martin, D.A.; Xie, L.; Taheri, R.; Lee, J.; Bolling, B.W. Aronia melanocarpa (chokeberry) polyphenol-rich extract improves antioxidant function and reduces total plasma cholesterol in apolipoprotein $\mathrm{E}$ knockout mice. Nutr. Res. 2013, 33, 406-413. [CrossRef] [PubMed]

2. Stanisavljević, N.; Samardžić, J.; Janković, T.; Šavikin, K.; Mojsin, M.; Topalović, V.; Stevanović, M. Antioxidant and antiproliferative activity of chokeberry juice phenolics during in vitro simulated digestion in the presence of food matrix. Food Chem. 2015, 175, 516-522. [CrossRef] [PubMed]

3. Denev, P.; Číž, M.; Kratchanova, M.; Blazheva, D. Black chokeberry (Aronia melanocarpa) polyphenols reveal different antioxidant, antimicrobial and neutrophil-modulating activities. Food Chem. 2019, 284, 108-117. [CrossRef] [PubMed]

4. Bhaswant, M.; Shafie, S.R.; Mathai, M.L.; Mouatt, P.; Brown, L. Anthocyanins in chokeberry and purple maize attenuate diet-induced metabolic syndrome in rats. Nutrition 2017, 41, 24-31. [CrossRef] [PubMed]

5. Thilavech, T.; Adisakwattana, S. Cyanidin-3-rutinoside acts as a natural inhibitor of intestinal lipid digestion and absorption. BMC Complement. Altern. Med. 2019, 19,1-10. [CrossRef] [PubMed]

6. Amin, F.U.; Shah, S.A.; Badshah, H.; Khan, M.; Kim, M.O. Anthocyanins encapsulated by PLGA@PEG nanoparticles potentially improved its free radical scavenging capabilities via $\mathrm{p} 38 / \mathrm{JNK}$ pathway against A $31-42$-induced oxidative stress. J. Nanobiotechnol. 2017, 15, 12. [CrossRef]

7. Wei, J.; Zhang, G.; Zhang, X.; Xu, D.; Gao, J.; Fan, J.; Zhou, Z. Anthocyanins from Black Chokeberry (Aroniamelanocarpa Elliot) Delayed Aging-Related Degenerative Changes of Brain. J. Agric. Food Chem. 2017, 65, 5973-5984. [CrossRef]

8. Faria, A.; Meireles, M.; Fernandes, I.; Santos-Buelga, C.; Gonzalez-Manzano, S.; Dueñas, M.; Freitas, V.; Mateus, N.; Calhau, C. Flavonoid metabolites transport across a human BBB model. Food Chem. 2014, 149, 190-196. [CrossRef]

9. Klisurova, D.; Petrova, I.; Ognyanov, M.; Georgiev, Y.; Kratchanova, M.; Denev, P. Co-pigmentation of black chokeberry (Aronia melanocarpa) anthocyanins with phenolic co-pigments and herbal extracts. Food Chem. 2019, 279, 162-170. [CrossRef]

10. Masters, C.L.; Bateman, R.; Blennow, K.; Rowe, C.C.; Sperling, R.A.; Cummings, J.L. Alzheimers disease. Nat. Rev. Dis. Primers 2015, 1, 15056. [CrossRef] 
11. Pacheco, S.M.; Soares, M.S.P.; Gutierres, J.M.; Gerzson, M.F.B.; Carvalho, F.B.; Azambuja, J.H.; Schetinger, M.R.C.; Stefanello, F.M.; Spanevello, R.M. Anthocyanins as a potential pharmacological agent to manage memory deficit, oxidative stress and alterations in ion pump activity induced by experimental sporadic dementia of Alzheimers type. J. Nutr. Biochem. 2018, 56, 193-204. [CrossRef] [PubMed]

12. Liu, X.; Hao, W.; Qin, Y.; Decker, Y.; Wang, X.; Burkart, M.; Schoetz, K.; Menger, M.D.; Fassbender, K.; Liu, Y. Long-term treatment with Ginkgo biloba extract EGb 761 improves symptoms and pathology in a transgenic mouse model of Alzheimer's disease. Brain Behav. Immun. 2015, 46, 121-131. [CrossRef] [PubMed]

13. Schimidt, H.L.; Garcia, A.; Martins, A.; Mello-Carpes, P.B.; Carpes, F.P. Green tea supplementation produces better neuroprotective effects than red and black tea in Alzheimer-like rat model. Food Res. Int. 2017, 100, 442-448. [CrossRef] [PubMed]

14. Ghosh, S.; Banerjee, S.; Sil, P.C. The beneficial role of curcumin on inflammation, diabetes and neurodegenerative disease: A recent update. Food Chem. Toxicol. 2015, 83, 111-124. [CrossRef]

15. Wen, H.; Fu, Z.; Wei, Y.; Zhang, X.; Ma, L.; Gu, L.; Li, J. Antioxidant Activity and Neuroprotective Activity of Stilbenoids in Rat Primary Cortex Neurons via the PI3K/Akt Signalling Pathway. Molecules 2018, 23, 2328. [CrossRef]

16. Andres-Lacueva, C.; Shukitt-Hale, B.; Galli, R.L.; Jauregui, O.; Lamuela-Raventos, R.M.; Joseph, J.A. Anthocyanins in aged blueberry-fed rats are found centrally and may enhance memory. Nutr. Neurosci. 2005, 8, 111-120. [CrossRef]

17. Hong, S.B.; Choi, J.H.; Chang, Y.K.; Mun, S. Production of high-purity fucose from the seaweed of Undaria pinnatifida through acid-hydrolysis and simulated-moving bed purification. Sep. Purif. Technol. 2019, 213, 133-141. [CrossRef]

18. Toumi, A.; Engell, S.; Diehl, M.; Bock, H.G.; Schlöder, J. Efficient optimization of simulated moving bed processes. Chem. Eng. Process. 2007, 46, 1067-1084. [CrossRef]

19. Strube, J.; Jupke, A.; Epping, A.; Schmidt-Traub, H.; Schulte, M.; Devant, R. Design, optimization, and operation of SMB chromatography in the production of enantiomerically pure pharmaceuticals. Chirality 1999, 11, 440-450. [CrossRef]

20. Wang, E.; Yin, Y.; Xu, C.; Liu, J. Isolation of high-purity anthocyanin mixtures and monomers from blueberries using combined chromatographic techniques. J. Chromatogr. A 2014, 1327, 39-48. [CrossRef]

21. Wojdyło, A.; Nowicka, P. Anticholinergic effects of Actinidia arguta fruits and their polyphenol content determined by liquid chromatography-photodiode array detector-quadrupole/time of flight-mass spectrometry (LC-MS-PDA-Q/TOF). Food Chem. 2019, 271, 216-223. [CrossRef]

22. Yang, J.; Wen, H.; Zhang, L.; Zhang, X.; Fu, Z.; Li, J. The influence of ripening stage and region on the chemical compounds in mulberry fruits (Morus atropurpurea Roxb.) based on UPLC-QTOF-MS. Food Res. Int. 2017, 100, 159-165. [CrossRef]

23. Mensor, L.L.; Menezes, F.S.; Leitão, G.G.; Reis, A.S.; Santos, T.C.; Coube, C.S.; Leitão, S.G. Screening of Brazilian plant extracts for antioxidant activity by the use of DPPH free radical method. Phytother. Res. 2001, 15, 127-130. [CrossRef]

24. Re, R.; Pellegrini, N.; Proteggente, A.; Pannala, A.; Yang, M.; Rice-Evans, C. Antioxidant activity applying an improved ABTS radical cation decolorization assay. Free Radical Bio. Med. 1999, 26, 1231-1237. [CrossRef]

25. Wen, M.; Ding, L.; Zhang, L.; Zhou, M.; Xu, J.; Wang, J.; Wang, Y.; Xue, C. DHA-PC and DHA-PS improved A $\beta 1-40$ induced cognitive deficiency uncoupled with an increase in brain DHA in rats. J. Funct. Foods 2016, 22, 417-430. [CrossRef]

26. Paxinos, G.; Watson, C. The Rat Brain in Stereotaxic Coordinates, 6th ed.; Elsevier Academic Press: New York, NY, USA, 2006.

27. Wen, M.; Xu, J.; Ding, L.; Zhang, L.; Du, L.; Wang, J.; Wang, Y.; Xue, C. Eicosapentaenoic acid-enriched phospholipids improve A $\beta 1-40$-induced cognitive deficiency in a rat model of Alzheimer's disease. J. Funct. Foods 2016, 24, 537-548. [CrossRef]

28. Sohanaki, H.; Baluchnejadmojarad, T.; Nikbakht, F.; Roghani, M. Pelargonidin improves memory deficit in amyloid $\beta 25-35$ rat model of Alzheimer's disease by inhibition of glial activation, cholinesterase, and oxidative stress. Biomed. Pharmacother. 2016, 83, 85-91. [CrossRef]

29. Chen, L.; Huang, J.; Yang, L.; Zeng, X.-A.; Zhang, Y.; Wang, X.; Chen, M.; Li, X.; Zhang, Y.; Zhang, M. Sleep deprivation accelerates the progression of alzheimer's disease by influencing A $\beta$-related metabolism. Neurosci. Lett. 2017, 650, 146-152. [CrossRef]

30. Lü, Y.-B.; Su, B.-G.; Yang, Y.-W.; Ren, Q.-L.; Wu, P.-D. Simulated moving bed separation of tocopherol homologues: Simulation and experiments. J. Zhejiang Univ. Sci. A 2009, 10, 758-766. [CrossRef]

31. Oszmianski, J.; Sapis, J.C. Anthocyanins in fruits of Aronia melanocarpa (chokeberry). J. Food Sci. 1988, 53, 1241-1242. [CrossRef]

32. Wang, S.; Liang, Y.; Zheng, S. Separation of epigallocatechin gallate from tea polyphenol by simulated moving bed chromatography. J. Chromatogr. A 2012, 1265, 46-51. [CrossRef] [PubMed]

33. Meng, L.; Li, B.; Li, D.; Wang, Y.; Lin, Y.; Meng, X.; Sun, X.; Liu, N. Cyanidin-3-O-glucoside attenuates amyloid-beta (A $\beta 1-$ 40)-induced oxidative stress and apoptosis in SH-SY5Y cells through a Nrf2 mechanism. J. Funct. Foods 2017, 38, 474-485. [CrossRef]

34. He, J.; Magnuson, B.A.; Giusti, M.M. Analysis of anthocyanins in rat intestinal contents-impact of anthocyanin chemical structure on fecal excretion. J. Agric. Food Chem. 2005, 53, 2859-2866. [CrossRef] [PubMed]

35. Miguel-Hidalgo, J.J.; Cacabelos, R. $\beta$-Amyloid (1-40)-induced neurodegeneration in the rat hippocampal neurons of the CA1 subfield. Acta Neuropathol. 1998, 95, 455-465. [CrossRef]

36. Miguel-Hidalgo, J.J.; Alvarez, X.A.; Cacabelos, R.; Quack, G. Neuroprotection by memantine against neurodegeneration induced by $\beta$-amyloid (1-40). Brain Res. 2002, 958, 210-221. [CrossRef]

37. Sarbishegi, M.; Heidari, Z.; Mahmoudzadeh-Sagheb, H.; Valizadeh, M.; Doostkami, M. Neuroprotective effects of Withania coagulans root extract on CA1 hippocampus following cerebral ischemia in rats. Avicenna J. Phytomed. 2016, 6, 399-409. 
38. Sun, X.; Xu, H.; Meng, X.; Qi, J.; Cui, Y.; Li, Y.; Zhang, H.; Xu, L. Potential use of hyperoxygenated solution as a treatment strategy for carbon monoxide poisoning. PLoS ONE 2013, 8, e81779. [CrossRef]

39. Kim, M.J.; Rehman, S.U.; Amin, F.U.; Kim, M.O. Enhanced neuroprotection of anthocyanin-loaded PEG-gold nanoparticles against A $\beta 1-42$-induced neuroinflammation and neurodegeneration via the NF-KB /JNK/GSK3 $\beta$ signaling pathway. Nanomed. NBM 2017, 13, 2533-2544. [CrossRef] 